\title{
Evidence for Two Mechanisms of Plasmid Transfer in Mixed Cultures of Staphylococcus aureus
}

\author{
By R. W. LACEY \\ Department of Pathology, King's Lynn General Hospital, \\ London Road, King's Lynn, Norfolk PE30 5QD
}

(Received 27 July 1979; revised 2 January 1980)

\begin{abstract}
Non-lysogenic Staphylococcus aureus strain 1030 was lysogenized with 12 different bacteriophages. Lysogeny was associated with acquisition of phage inducibility by mitomycin $\mathrm{C}$ treatment or ultraviolet irradiation, with the presence of plaque-forming phage in culture supernatants and with considerable narrowing in susceptibility to the typing bacteriophages and also with increased sensitivity to trimethoprim and sulphadiazine. The presence of prophages in the donor and/or the recipient could either promote or inhibit transfer of plasmids between mixed cultures. Transfer frequencies in mixed culture after $18 \mathrm{~h}$ incubation could be as high as $7 \cdot 0 \times 10^{-1}$ resistant recipients/final donor, and evidence was adduced for a mechanism distinct from transfer by spontaneous transduction. It is suggested that this method of gene transfer be described as 'phage-mediated conjugation'. Chromosomal genes were not transferred by this method. Two similar plasmids $c p-2$ and $c p-3$ were able to promote their own transfer through clones of 1030 ; plasmids coding for resistance to either neomycin or tetracycline could be transferred to a recipient by the presence of $c p-2$ or $c p-3$ simultaneously in the donor. The presence of plasmids in 1030 was associated with a small increase in sensitivity to trimethoprim or sulphadiazine.
\end{abstract}

\section{INTRODUCTION}

Bacteriophages extracted from cultures of Staphylococcus aureus are well known to mediate generalized transduction within this species (Pattee \& Baldwin, 1961; Novick, 1963). The possibility that antibiotic resistance in $S$. aureus might spread between isolates in nature by transduction was strengthened by the construction of an element that contained DNA of both plasmid and phage origin (Novick, 1967). This element could pass spontaneously between cells in mixed cultures in vitro at frequencies of about $10^{-4}$, and at rather lower frequencies following inoculation of the cultures into mice (Novick \& Morse, 1967). However, a specific helper phage in the donor is necessary for the production of transferring particles, and the role that such an element can play in the spread of antibiotic resistance in staphylococci in general is therefore uncertain. Spread of resistance by generalized transduction in nature has also been considered unlikely to occur commonly because of the low frequencies attainable in vitro, typically $10^{-6}$ to $10^{-8}$ (Richmond, 1969). However, resistance to several antibiotics can be transferred spontaneously from unmanipulated cultures to appropriate recipients in vitro, and experimentally on the skin surface (Lacey, 1971 $a, b$, 1975). This transfer requires between 0.001 and $0.01 \mathrm{M}-\mathrm{Ca}^{2+}$ or $\mathrm{Mg}^{2+}$, is abolished by sodium citrate and requires the use of a phage-resistant recipient; however, the frequency increases with high cell density, and transferring particles may not be detectable in the donor culture supernatant (Lacey, 1971 $a$; Lacey \& Stokes, 1979). Whilst such transfer might be mediated via generalized transduction, with the phages being either cell-bound or 
unstable (Lacey, 1975), there are some inconsistencies with this proposal. (1) Witte (1977) has shown that transfer of antibiotic resistance in mixed culture can occur to an encapsulated recipient strain, whilst a cell-free extract containing 'transducing' phages cannot mediate transfer to such a culture. (2) Lantos (1977) has shown that a single resistance determinant is transferred from a donor to a recipient by conventional transduction, but following incubation of whole cells of the donor with the recipient, three resistance determinants are transferred simultaneously. (3) Naidoo \& Noble (1978) have shown that gentamicin resistance can be transferred between strains when they were inoculated on to the skin of human volunteers or mice; however, transfer of gentamicin resistance between these cultures was not detected when they were cultured in nutrient broth. (4) The frequency of transfer of drug resistance between strains in mixed culture may increase when the recipient (1030) is lysogenic for a prophage (Lacey, 1979).

The role of transformation in mediating gene transfer between staphylococci in vivo is not clear because to obtain the optimal frequency of transformation a concentration of $0 \cdot 1 \mathrm{M}-\mathrm{Ca}^{2+}$ is required and extracellular DNAase must be inhibited. Bacteriophage gene(s) in the recipient appear to be essential for transformation (Lindberg \& Novick, 1973).

Thus, bacteriophages appear to be closely involved in gene transfer in $S$. aureus in vitro, although their precise role(s) is uncertain in vivo. This paper describes experiments which show that bacteriophage carriage in $S$. aureus 1030 can either promote or inhibit plasmid transfer.

\section{METHODS}

Media. Nutrient broth (Oxoid no. 2) contained additional $0.01 \mathrm{M}-\mathrm{CaCl}_{2}$ unless otherwise stated. Nutrient agar was obtained from Mast Laboratories, Liverpool, and diagnostic sensitivity test (DST) agar from Oxoid. Milk agar was obtained by the addition of 'Ideal' milk to Mast agar to a final concentration of $30 \%(\mathrm{v} / \mathrm{v})$.

Antibiotic sensitivity. Sensitivity was determined by the method of Lacey (1979) except that sulphadiazine and trimethoprim were incorporated into DST agar at the following concentrations: $0.05,0.075,0 \cdot 10,0 \cdot 15$, $0 \cdot 20,0 \cdot 25,0 \cdot 30,0 \cdot 35,0 \cdot 4,0 \cdot 5,1 \cdot 0 \mathrm{mg}$ trimethoprim $1^{-1} ; 5,7 \cdot 5,10,20,25,30,40,50 \mathrm{mg}$ sulphadiazine $1^{-1}$.

Bacteriophage typing, mitomycin C induction, ultraviolet light induction, and transduction. These methods have been described previously (Lacey, 1971 $a$, 1979). The optimal mitomycin $\mathrm{C}$ concentration for induction of lysogenic derivatives of strain 1030 was $1.0 \mathrm{mg} \mathrm{1}^{-1}$. Accordingly, to test for inducibility of derivatives of strain 1030 , they were treated with either 1.0 or $3.0 \mathrm{mg}$ mitomycin $\mathrm{Cl}^{-1}$. Induction with either mitomycin $\mathrm{C}$ or ultraviolet light was assessed as clearing relative to that of untreated cultures: ++ denoted complete clearing, + a reduction in turbidity to about $0 \cdot 2$, and \pm a reduction in turbidity to between $0 \cdot 2$ and $0 \cdot 5$. During transduction, care was taken to protect recipients from becoming lysogenic by using a phage to cell ratio of 0.01 , and after allowing $15 \mathrm{~min}$ for phage adsorption in the presence of $0.01 \mathrm{M}$-calcium chloride, all subsequent procedures were done in the presence of $0.02 \mathrm{M}$-sodium citrate. Phages were titrated on culture 1030, unless otherwise stated. Transduction frequencies were expressed as the ratio of the number of transductants to the number of cells in the donor culture at the time of addition of mitomycin $\mathrm{C}$. Phages were checked for sterility following filtration through $0.45 \mu \mathrm{m}$ Millipore filters and titrated on strain 1030 wild.

Staphylococcus aureus strain. Strain 1030 was obtained in 1970 from Dr R. P. Novick, New York, and maintained on nutrient agar slopes at room temperature for 8 years with one subculture. This culture is described as non-lysogenic (Novick, 1967), is lysed at varying efficiencies by each of the currently used typing phages and is non-inducible with ultraviolet light or mitomycin C. Strain 1030 was lysogenized by several typing phages and one of the prophages harboured by strain 13136 (Lacey, 1972). The latter is designated C. Lysogens were studied for sensitivity to trimethoprim and sulphadiazine because thymine (or thymidine) starvation invoked by these agents results in induction of lysogenic cultures (Melechen, 1962; Devoret \& Blanco, 1970). Filtrates of this culture that had been treated with 1.0 or $3.0 \mathrm{mg}$ mitomycin $\mathrm{C}^{-1}$, together with a lysogenic culture derived from this $[1030(\mathrm{C})]$, were examined under the electron microscope by Evelyn Lewis and Dr P. Bennett by the method of Robinson et al. (1977). The 1030(C) lysate contained phage particles, but neither preparation of 1030 wild strain contained either intact phage particles or structures resembling phage components. Filtrates of culture 1030 were also studied for plaque-forming activity on 50 freshly isolated clinical strains of $S$. aureus. No plaques were seen. It is inferred that culture 1030 is extremely unlikely to carry complete prophages. Since culture 1030 was to be manipulated, a chromosomal mutant (resistant to $100 \mathrm{mg}$ rifampicin $\mathrm{1}^{-1}$ ) was selected initially; all derivatives of 1030 also showed a 


\section{Table 1. Staphylococcal plasmids}

Plasmid

tet-r
neo-r
chl-r
pen-r
cad-r
cpns
$c p-1$
$c p-2$
$c p-3$
sn

Resistance(s) coded for

Tetracycline
Neomycin
Chloramphenicol
Penicillin, cadmium, arsenate and mercury
Cadmium, arsenate and mercury
Penicillin, cadmium, neomycin, streptomycin
Penicillin and cadmium
Penicillin and cadmium
Penicillin and cadmium
Streptomycin and neomycin

\section{Reference}

Lacey \& Grinsted (1973)

Chopra et al. (1973)

Chopra et al. (1973)

Lacey \& Grinsted (1973)

Chopra et al. (1973)

Lacey (1979)

Lacey (1979)

Lacey (1979)

Lacey (1979)

Lacey (1979)

characteristic very pale buff pigmentation on milk agar and produced deoxyribonuclease (tested on Oxoid DNAase agar).

Plasmids. Except for plasmid cpns and four derivatives, $c p-1, c p-2, c p-3$ and $s n$ (Lacey, 1979), the plasmids used have been fully characterized (Table 1).

Nomenclature. Prophage carriage is denoted by the corresponding arabic number in parenthesis, genes of plasmid origin in italics, and mutants selected for chromosomal resistance by capital letters (S, streptomycin; F, fusidic acid). All cultures were rifampicin-resistant; this is not specified for each derivative. Culture 1030(55)tet-r is 1030 lysogenic for typing phage 55 and contains a plasmid determining tetracycline resistance, and $1030(\mathrm{C}) \mathrm{S}$ is lysogenic for phage $\mathrm{C}$ (from strain 13136) and possesses chromosomal resistance to streptomycin $\left(100 \mathrm{mg} \mathrm{l}^{-1}\right)$.

Transfer of resistance in mixed cultures. This was done by the method of Lacey $(1971 \mathrm{a})$; modifications are described where appropriate. Unless otherwise stated, the frequency of transfer is defined as the ratio of the number of resistant recipients to the final number of donors after $18 \mathrm{~h}$ incubation. From the detailed study of transfer of tet-r to and from clones of 1030(55) (see below) it is assumed that the transfer frequencies represent predominantly transfer from the putative donor to recipient.

Resistance transfer. This was detected by the incorporation of either $4 \mathrm{mg}$ tetracycline $1^{-1}, 10^{-4} \mathrm{M}$-cadmium acetate, $5 \mathrm{mg}$ neomycin $1^{-1}$ or $25 \mathrm{mg}$ chloramphenicol base $\mathrm{1}^{-1}$ with either 100 or $400 \mathrm{mg}$ streptomycin $1^{-1}$ or $10 \mathrm{mg}$ fusidic acid $1^{-1}$. Coincidental transfer of two resistance markers was studied using nutrient agar containing agents at $70 \%$ of these concentrations or by replica-plating of colonies selected for acquisition of a single resistance.

Storage of cultures. Purified clones of each derivative were stored on the surface of nutrient agar plates at $4{ }^{\circ} \mathrm{C}$ for up to 2 months when each was subcultured.

Transfer experiments under anaerobic conditions. Broth cultures were mixed on the surface of nutrient agar containing $0.01 \mathrm{M}-\mathrm{Ca}^{2+}$ that had been cooled to $4{ }^{\circ} \mathrm{C}$ and were incubated in a Baird \& Tatlock jar in an atmosphere of $10 \% \mathrm{CO}_{2}$ and $90 \% \mathrm{H}_{2}$. Controls included known obligate aerobic and anaerobic cultures in each jar. Some of the plates inoculated with the two cultures were sampled after $1 \mathrm{~h}$ incubation to exclude the possibility that transfer had occurred during the seeding of the plates. After incubation for $20 \mathrm{~h}$ at $37^{\circ} \mathrm{C}$, the plates were withdrawn from the jar and flooded with $2.0 \mathrm{ml} 0.05 \mathrm{M}$-sodium citrate, and bacteria were emulsified and diluted in water containing $0.02 \mathrm{M}$-sodium citrate, before viable counts were performed on appropriate media also containing $0.02 \mathrm{M}$-sodium citrate.

Chemicals. Bovine pancreatic deoxyribonuclease I was added to cultures at 1 to $100 \mathrm{mg} 1^{-1}$, bovine spleen deoxyribonuclease II at $10 \mathrm{mg}$ to $1 \mathrm{~g} \mathrm{l}^{-1}$, and micrococcal nuclease at 0.1 to $10 \mathrm{mg}^{-1}$. These enzymes and mitomycin $\mathrm{C}$ were obtained from Sigma.

\section{RESULTS}

\section{Effect of plasmid carriage on the properties of strain 1030}

Plasmid genes were transduced individually into strain 1030 wild using mitomycin Cinduced lysates of donor cultures; during the construction of multiresistant clones of strain 1030 by successive transduction there was no evidence for incompatibility between the plasmids. All the plasmid-containing derivatives had the same bacteriophage typing pattern as that of strain 1030 wild, and all except $1030 \mathrm{cp}-2$, and $1030 \mathrm{cp}-3$ were non-inducible with mitomycin $\mathrm{C}$ or ultraviolet light. However, broth culture filtrates of $1030 c p-2$ and $1030 c p-3$ 
Table 2. Sensitivity of strain 1030 and derivatives to sulphadiazine and trimethoprim

$\begin{array}{lcc} & \text { Minimum inhibitory concn }\left(\mathrm{mg} \mathrm{l}^{-1}\right)^{*} \\ \text { Strain } & \text { Sulphadiazine } & \text { Trimethoprim } \\ 1030 \text { wild } & 50 & 0 \cdot 40 \\ 1030 \text { tet }-r & 30 & 0 \cdot 25 \\ 1030 \text { neo-r } & 25 & 0 \cdot 30 \\ 1030 \text { chl-r } & 30 & 0 \cdot 25 \\ 1030 \text { pen-r } & 30 & 0 \cdot 30 \\ 1030 \text { cad-r } & 30 & 0 \cdot 25 \\ 1030 \text { cpns } & 30 & 0 \cdot 20 \\ 1030 \text { cp }-1 & 25 & 0 \cdot 25 \\ 1030 \text { cp }-2 & 30 & 0 \cdot 20 \\ 1030 \text { cp }-3 & 25 & 0 \cdot 20 \\ 1030 \text { sn } & 30 & 0 \cdot 30 \\ 1030(C) & 15 & 0 \cdot 20 \\ 1030(52) & 15 & 0 \cdot 20 \\ 1030(79) & 15 & 0 \cdot 15 \\ 1030(55) & 15 & 0 \cdot 20 \\ 1030(71) & 7 \cdot 5 & 0 \cdot 15 \\ 1030(95) & 20 & 0 \cdot 25 \\ 1030(42 \mathrm{E}) & 15 & 0 \cdot 15 \\ 1030(54) & 20 & 0 \cdot 20 \\ 1030(77) & 20 & 0 \cdot 25 \\ 1030(81) & 20 & 0 \cdot 25 \\ 1030(88) & 10 & 0 \cdot 15 \\ 1030(96) & 15 & 0 \cdot 25 \\ 105 \text { r }) & & \end{array}$

* Similar results were obtained on at least three occasions.

did not contain any plaque-forming units (p.f.u.), and neither did the other derivatives. Thus, except for culture 1030cp-2 and 1030cp-3 (and see below), there was no evidence for lysogeny in these cultures. However, all were reproducibly slightly more sensitive to trimethoprim and sulphadiazine than culture 1030 wild (Table 2).

Effect of lysogenization on the properties of strain 1030

Twelve lysogenic derivatives of strain 1030 were established; these were separately lysogenic for phage $C$ (from strain 13136) and typing phages 52, 79, 81, 55, 71, 95, 42E, 54, 77,88 and 96. Each lysogen showed a reduction in susceptibility to several typing phages (Table 3) and increased sensitivity to trimethoprim and sulphadiazine (Table 2). All except strain $1030(52)$ were fully inducible [i.e. cultures completely cleared and contained $>10^{5}$ p.f.u. $\mathrm{ml}^{-1}$ ] with both mitomycin $\mathrm{C}$ and ultraviolet light induction. Non-inducible cultures contained $<10^{4}$ p.f.u. $\mathrm{ml}^{-1}$. Culture $1030(52)$ cleared slightly $( \pm)$ after mitomycin $\mathrm{C}$ treatment, but not after treatment with ultraviolet light. However, the culture supernatants of purified 1030(52) contained plaque-forming agents; lysogeny in this derivative is therefore established. A proportion (17\%) of 1030(55), 1030(88) and 1030(52) that had been selected with low concentrations of trimethoprim $\left(0.25\right.$ to $\left.0.30 \mathrm{mg} \mathrm{l}^{-1}\right)$ had regained the complete typing pattern of 1030 wild. It is inferred that exposure of lysogenic cultures to levels of trimethoprim just above those that are inhibitory to the majority of the culture selects cells that are non-lysogenic.

\section{Transduction of tetracycline resistance and penicillinase production from strain 13136 to 1030}

A mitomycin C-induced lysate of 13136 was used to transduce both tetracycline resistance and penicillinase production to culture 1030 wild and its lysogenic derivatives. Transduction was successful to each recipient except 1030(52). Replica-plating of at least 50 transductants obtained from each recipient showed no cotransduction of pen-r and tet-r. Mixed culture 


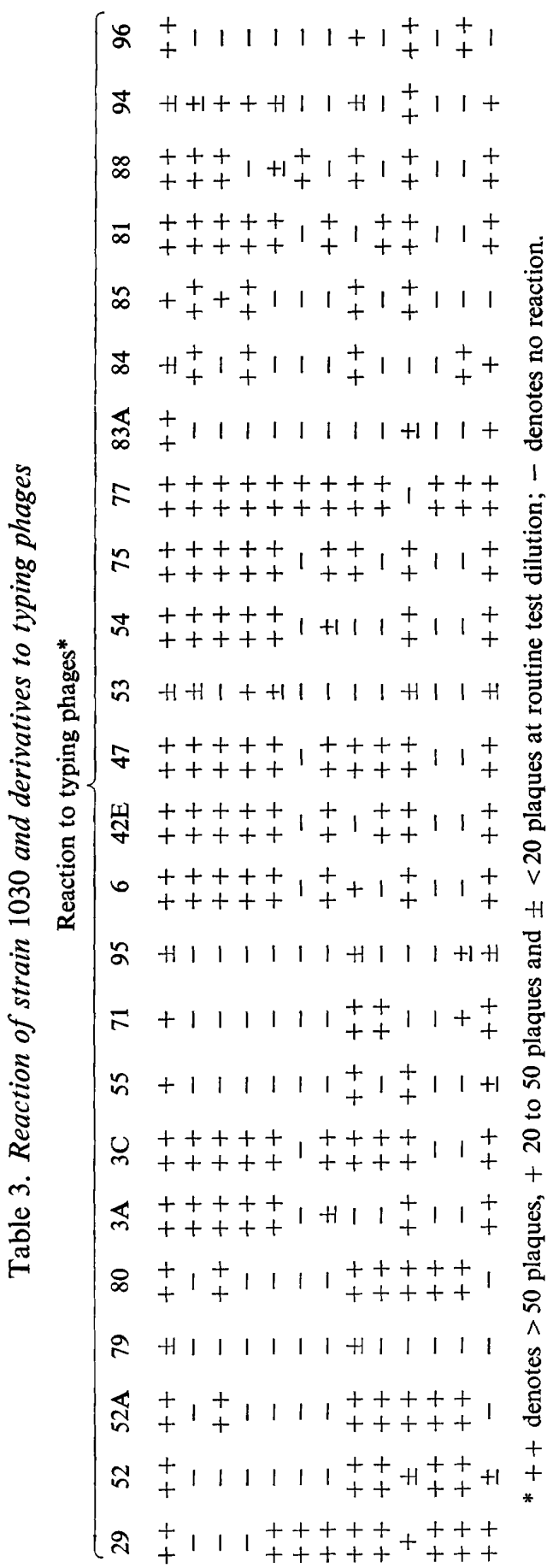

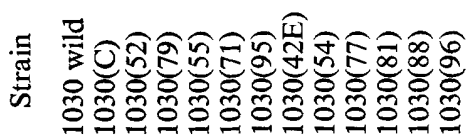


Table 4. Transfer of tet-r and pen-r from strain 13136 to 1030 by transduction and by mixed culture incubation

Note that the transfer frequencies from cell-free lysates are derived differently to those from mixed cultures, and the two are not comparable.

\begin{tabular}{|c|c|c|c|c|}
\hline \multirow[b]{2}{*}{ Recipient } & \multicolumn{2}{|c|}{$\begin{array}{l}\text { Frequency of transduction } \\
\text { with cell-free lysate* }\end{array}$} & \multicolumn{2}{|c|}{$\begin{array}{l}\text { Frequency of transfer } \\
\text { in mixed culture } †\end{array}$} \\
\hline & tet-r & $p e n-r$ & tet-r & $p e n-r$ \\
\hline 1030 wild & $1.3 \times 10^{-4}$ & $7 \cdot 7 \times 10^{-6}$ & $1.0 \times 10^{-5}$ & $9 \cdot 1 \times 10^{-8}$ \\
\hline $1030(\mathrm{C})$ & $9.7 \times 10^{-5}$ & $4.6 \times 10^{-7}$ & $2.8 \times 10^{-6}$ & $8.3 \times 10^{-8}$ \\
\hline $1030(52)$ & $0 \ddagger$ & 0 & 0 & 0 \\
\hline 1030(79) & $5.0 \times 10^{-4}$ & $1 \cdot 1 \times 10^{-6}$ & $4.9 \times 10^{-6}$ & $2.9 \times 10^{-8}$ \\
\hline $1030(55)$ & $6.1 \times 10^{-5}$ & $5.6 \times 10^{-7}$ & $7 \cdot 7 \times 10^{-6}$ & $1.8 \times 10^{-8}$ \\
\hline $1030(71)$ & $1.0 \times 10^{-5}$ & $3.9 \times 10^{-7}$ & $4.2 \times 10^{-5}$ & $1.7 \times 10^{-8}$ \\
\hline $1030(95)$ & $7 \cdot 1 \times 10^{-4}$ & $2.0 \times 10^{-6}$ & $3.7 \times 10^{-5}$ & $1.4 \times 10^{-7}$ \\
\hline $1030(42 \mathrm{E})$ & $2.4 \times 10^{-4}$ & $3.9 \times 10^{-7}$ & $1.0 \times 10^{-5}$ & $2.4 \times 10^{-8}$ \\
\hline $1030(54)$ & $2.1 \times 10^{-4}$ & $9.8 \times 10^{-7}$ & $2 \cdot 2 \times 10^{-5}$ & $1.3 \times 10^{-8}$ \\
\hline $1030(77)$ & $9.1 \times 10^{-5}$ & $2.4 \times 10^{-6}$ & $8.8 \times 10^{-6}$ & $7 \cdot 3 \times 10^{-8}$ \\
\hline $1030(81)$ & $1.7 \times 10^{-4}$ & $4.3 \times 10^{-6}$ & $2.4 \times 10^{-6}$ & $7.3 \times 10^{-8}$ \\
\hline $1030(88)$ & $9.0 \times 10^{-5}$ & $8.2 \times 10^{-7}$ & $9.9 \times 10^{-6}$ & $1.0 \times 10^{-7}$ \\
\hline $1030(96)$ & $5.0 \times 10^{-5}$ & $8.0 \times 10^{-7}$ & $5 \cdot 1 \times 10^{-6}$ & $3.5 \times 10^{-8}$ \\
\hline
\end{tabular}

* No. of resistant recipients/initial no. of donors at induction.

$\dagger$ No. of resistant recipients/final no. of donors.

$\ddagger<10^{-10}$.

incubation also showed transfer of either pen-r or tet-r separately to each recipient except to $1030(52) \mathrm{S}$ (Table 4). Failure to transfer pen-r or tet-r to 1030(52) might be due to failure of phage adsorption to this strain since this culture is resistant to lysis by phage $\mathrm{C}$. To test for this, $10^{6}$ p.f.u. $\mathrm{ml}^{-1}$ of phages $\mathrm{C}, 29,55$ and 77 were incubated with 1030 wild and $1030(52)$ for $60 \mathrm{~min}$ and then the cultures were filtered. Culture 1030 wild adsorbed more than $99 \%$ of each phage; $1030(52)$ adsorbed phage 77 by more than $99 \%$, but failed to adsorb phages C, 29 and 55 (the titre reducing by less than $10 \%$ in each case). Possibly lysogeny with phage 52 prevents phage adsorption, although it could be that $1030(52)$ is both lysogenic and a phage-resistant mutant.

Thus, the properties of transfer of pen-r and tet-r from strain 13136 to 1030 by transduction and mixed culture incubation were similar - the mechanism of mixed culture transfer between 13136 and 1030 is therefore probably by spontaneous transduction. Consistent with this was the observation that when the transductants, obtained after mixed culture incubation, were subcultured to nutrient agar containing $0.01 \mathrm{M}-\mathrm{CaCl}_{2}$, spontaneous phage activity was seen in seven resistant recipients obtained by either method. In contrast, spontaneous phage activity was uncommon following transfer between donors and recipients both derived from 1030 (see below). Culture filtrates of 13136 were also able to transfer tet-r $\left.\left[1 \cdot 5 \times 10^{1} \text { resistant recipients (ml filtrate) }\right)^{-1}\right]$ and pen-r $\left[1 \cdot 0 \times 10^{2} 1^{-1}\right]$ to 1030 wild.

These findings also show that except for 1030(52), which was not evaluated, prophage carriage does not affect the establishment of either the pen-r or tet-r plasmids individually.

Gene transfer mediated by the addition of typing phages in mixed culture experiment

The prospective donor, 1030tet-r, appears to be non-lysogenic and did not transfer tetracycline resistance to recipient 1030S in mixed broth cultures. Mixed cultures were then prepared, with appropriate controls, containing $10^{7}$ each of donor and recipient cells $\mathrm{ml}^{-1}$ and $10^{5}$ p.f.u. $\mathrm{ml}^{-1}$ of one of the typing phages or phage C. After $24 \mathrm{~h}$ incubation, the mixtures were diluted and inoculated on to antibiotic-containing nutrient agar plates to 
Table 5. Transfer of resistance between cultures 1030 tet-r and $1030 \mathrm{~S}$ mediated by bacteriophages

$\begin{array}{cccc}\text { Note that the direction of transfer is not established. } & \begin{array}{c}\text { No. of cells } \mathrm{ml}^{-1} \\ \text { Nove added } \\ \text { having phenotype } \\ 1030 \text { Stet }-r\end{array} & \begin{array}{c}\text { Po. of cells } \mathrm{ml}^{-1} \\ \text { having phenotype } \\ 1030 \text { Stet }-r\end{array} \\ 29 & 8 \cdot 4 \times 10^{3} & 47 & 0 \\ 52 & 0^{*} & 53 & 0 \\ 52 \mathrm{~A} & 3 \cdot 0 \times 10^{2} & 54 & 0 \\ 79 & 2 \cdot 0 \times 10^{3} & 75 & 0 \\ 80 & 0 & 77 & 0 \\ 3 \mathrm{~A} & 0 & 83 \mathrm{~A} & 5 \cdot 4 \times 10^{2} \\ 3 \mathrm{C} & 0 & 84 & 0 \\ 55 & 7 \cdot 2 \times 10^{3} & 85 & 5 \cdot 9 \times 10^{3} \\ 71 & 7 \cdot 5 \times 10^{4} & 88 & 3 \cdot 0 \times 10^{2} \\ 95 & 4 \cdot 2 \times 10^{4} & 81 & 0 \\ 6 & 0 & 94 & 0 \\ 42 \mathrm{E} & 0 & 96 & 8 \cdot 6 \times 10^{4}\end{array}$

$*<10^{1} \mathrm{ml}^{-1}$; the mutation frequency of 1030 tet-r to 1030 Stet- $r$ was always $<10^{-9}$, i.e. there were always $<10^{1}$ such derivatives in control cultures.

select for transfer of resistance. Derivatives resistant to both tetracycline and streptomycin were obtained from cultures to which the following phages had been added: 29, 52A, 79, $55,71,95,83 \mathrm{~A}, 85,88$ and 96 (Table 5). The frequency of acquired resistance/total bacteria varied from about $10^{-3}$ to $10^{-6}$. These results indicate that many phages can be involved in gene transfer, although it is not possible to ascertain the mechanism from these experiments.

\section{Effect of prophage carriage in donor and recipient on transfer of tetracycline resistance in mixed culture}

Culture 1030 and nine lysogenic derivatives each containing a plasmid coding for tetracycline resistance were incubated with each of the 13 recipients, 12 being lysogenic. Transfer of resistance occurred in most mixtures, but at variable frequencies. Typical results are shown in Table 6 . The presence of phage $C$ or 52 in the recipient was usually associated with a low frequency of transfer (about $10^{-7}$ ) whilst little transfer was seen when $1030(42 \mathrm{E})$ tet-r was incubated with each recipient. Although the direction of the plasmid transfer was not absolutely certain, these results suggest that prophage in either the donor or the recipient may permit transfer.

The effect of lysogeny in the donor was first studied in more detail. Donor cultures 1030 tet-r, 1030neo-r, 1030 chl-r and nine cultures of each lysogenic derivative of 1030 were incubated singly with the non-lysogenic recipient (1030S). As expected, no transfer occurred from 1030tet- $r$, 1030neo- $r$ or 1030 chl-r to non-lysogenic 1030S. Following incubation of the lysogenic donors with the putative recipients, plasmid transfer varied from about $10^{-8}$, for the putative donors lysogenic for phage $42 \mathrm{E}$, to $10^{-3}$, for cultures lysogenic for phage 95 . Examination of a proportion of the resistant recipients obtained after mixed culture incubation revealed that all still gave the intact typing pattern of 1030 wild and their culture filtrates did not produce plaques on culture 1030 wild. Thus, there was no evidence for plasmid transfer mediated by first the passage of the phage from the donor to the recipient, followed by transfer of the plasmid coding for antibiotic resistance from the donor to a lysogenic recipient. It is probable, however, that the direction of plasmid transfer was from the putative donors to the recipients.

\section{Transfer of tetracycline resistance from strain 1030tet-r to 1030(55)SF}

The effect of lysogeny in the recipient 1030(55) was studied in detail since it had apparently been found to acquire resistance at high frequency (Table 6). To establish that the direction 


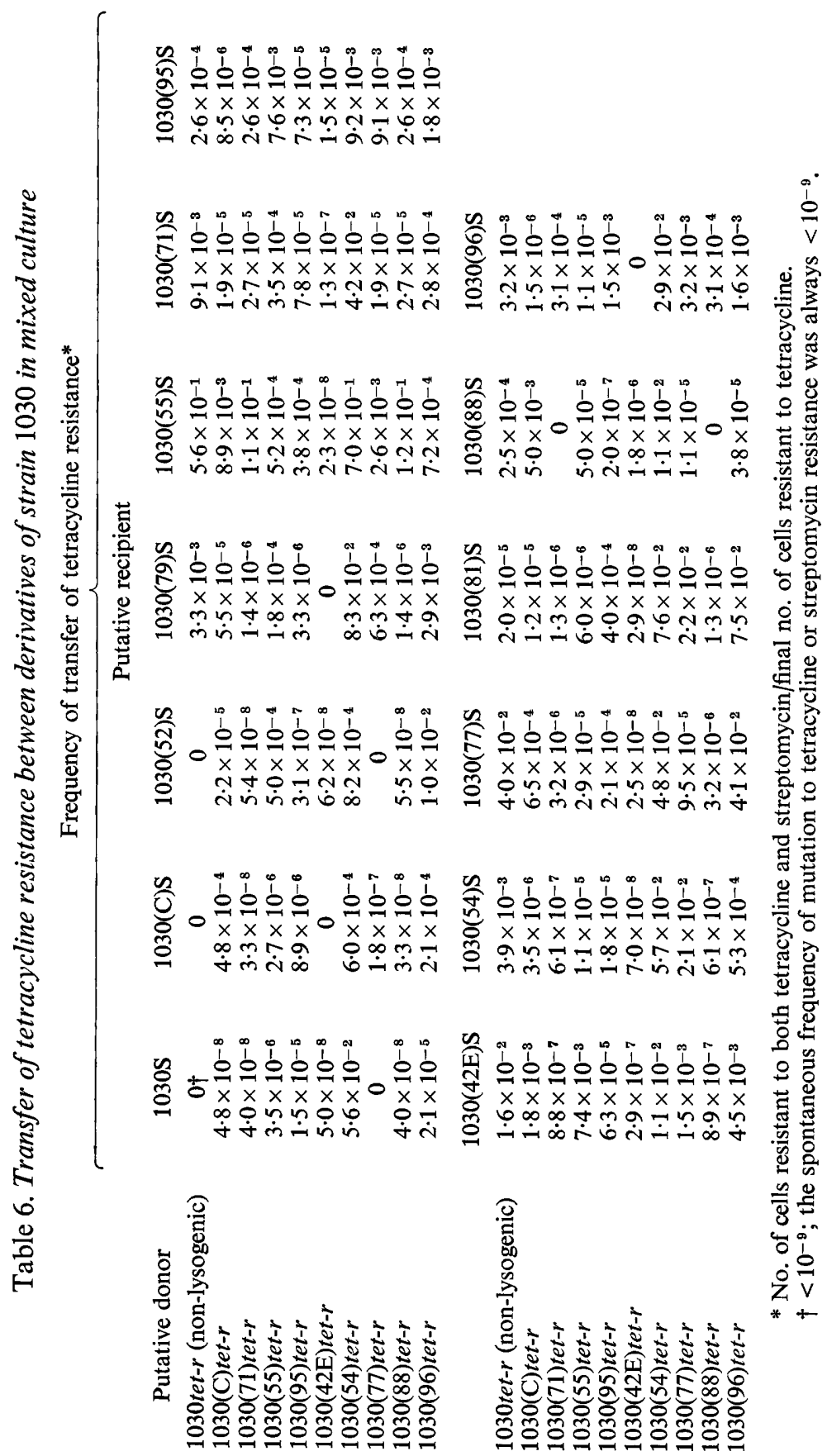


Table 7. Effect of carriage of phage 55 on the transfer of tetracycline resistance between clones of 1030

\begin{tabular}{|c|c|c|c|}
\hline \multirow[b]{2}{*}{ Donor } & \multirow[b]{2}{*}{ Recipient } & \multicolumn{2}{|c|}{$\begin{array}{l}\text { Transfer frequency* } \\
\text { (duplicate expts) }\end{array}$} \\
\hline & & & \\
\hline 1030tet-r & 1030SF & 0 & 0 \\
\hline 1030tet-r & $1030(55) \mathrm{SF}$ & $3 \cdot 0 \times 10^{-1}$ & $7 \cdot 3 \times 10^{-1}$ \\
\hline $1030(55)$ tet $-r$ & $1030 \mathrm{SF}$ & $2.5 \times 10^{-6}$ & $6.5 \times 10^{-6}$ \\
\hline $1030(55)$ tet $-r$ & $1030(55) \mathrm{SF}$ & $6.0 \times 10^{-5}$ & $4.5 \times 10^{-5}$ \\
\hline
\end{tabular}

of plasmid transfer was from the non-lysogenic culture 1030 tet- $r$ to culture $1030(55) \mathrm{S}$, a mutant of the latter was isolated with an additional chromosomal marker (resistance to fusidic acid, F). The loci of these two markers were widely separated since there was no cotransduction of the resistances, using phage 88 as vector (data not shown). Cultures of 1030tet-r and 1030(55)SF were mixed in mid-exponertial phase and, with controls, sampled at $5,10,15,20$ and $30 \mathrm{~min}$ and $1,2,4,6$ and $20 \mathrm{~h}$ by plating undiluted and diluted cultures on to nutrient agar containing $4.0 \mathrm{mg}$ tetracycline $\mathrm{l}^{-1}, 100 \mathrm{mg}$ streptomycin $\mathrm{l}^{-1}$ and $0.02 \mathrm{M}$ sodium citrate. Transfer of resistance was first detected after 20 min co-incubation. Forty of these derivatives were examined in detail ; all possessed the phenotype 1030(55)SFtet-r including the phage typing pattern of $1030(55)$ and were lysogenic for a phage resembling 55 in its spectrum of lysis. The rapid appearance of these cells argued that transfer was not mediated by phage 55 released from the recipient and propagated on the donor before conveyance of the tet-r plasmid back to the recipient. Filtrates of broth cultures of 1030(55)SF contained phages lytic for 1030tet-r although little spontaneous phage activity was seen after application of the mixed culture to the surface of nutrient agar containing $0 \cdot 01 \mathrm{M}-\mathrm{CaCl}_{2}$. Furthermore, filtrates of the mixed culture after $4 \mathrm{~h}$ incubation could not transfer tetracycline resistance to 1030 wild. It is inferred that transfer of tetracycline resistance is in the direction from 1030tet-r to $1030(55) \mathrm{SF}$. The frequency (expressed as resistant recipients/donors) increased from about $10^{-7}$ at $20 \mathrm{~min}$ to $3.0 \times 10^{-3}$ at $4 \mathrm{~h}$, $3.6 \times 10^{-2}$ at $6 \mathrm{~h}$ and to $1.2 \times 10^{-1}$ or $7.0 \times 10^{-1}$ in replicate experiments at $20 \mathrm{~h}$. Transfer (approximate frequency $10^{-2}$ at $20 \mathrm{~h}$ ) also occurred on the surface of nutrient agar incubated anaerobically, with precautions taken to exclude transfer during processing (see Methods). The addition of mammalian or micrococcal nucleases had no effect on the transfer under aerobic conditions. The optimal molarity of $\mathrm{Ca}^{2+}$ and $\mathrm{Mg}^{2+}$ for transfer was between 0.001 and $0.025 \mathrm{M}$, and transfer was abolished by the addition of $0.01 \mathrm{M}$-sodium citrate to nutrient broth (this contained $0.5 \mathrm{mM}-\mathrm{CaCl}_{2}$ ). The effect of cell density on transfer was investigated by altering the oxygen tension during incubation (Lacey \& Stokes, 1979). The transfer frequency varied from about $3 \times 10^{-1}$ at a final cell density of $10^{9}$ colony-forming units (c.f.u.) $\mathrm{ml}^{-1}$ to $6 \times 10^{-3}$ at a density of $10^{7}$ c.f.u. $\mathrm{ml}^{-1}$.

\section{Effect of prophage 55 in strain 1030tet-r on transfer frequency}

Culture 1030(55)tet-r was incubated with 1030SF or 1030(55)SF. Transfer was detected after $30 \mathrm{~min}$, and in each mixture the frequency increased until $20 \mathrm{~h}$, although the final frequencies were less than those obtained for 1030tet-r and 1030(55)SF (Table 7). Examination of 20 derivatives from each mixture showed drug sensitivity and lysogeny consistent with a direction of transfer from the tetracycline-resistant culture to the putative recipient. Thus, the presence of phage 55 in the donor can itself promote transfer although at rather low frequency, but its presence in the donor inhibits transfer to a recipient that also harbours this phage. 
Transfer of plasmids from the non-lysogenic donor 1030 to recipients derived from 1030

Non-lysogenic donors 1030neo-r, 1030chl-r, 1030cpns, 1030pen-r, 1030cad-r and 1030cp-1 could not transfer resistance (frequency $<10^{-9}$ ) to the non-lysogenic recipient $1030 \mathrm{~S}$ in mixed culture. Transfer to the other lysogenic derivatives of 1030 occurred at variable frequency; the plasmids from 1030cpns, 1030cad-r and 1030pen-r transferred at frequencies from $10^{-4}$ to $10^{-8}$, the plasmids from 1030neo-r and $1030 \mathrm{chl}-r$ at frequencies from $1.5 \times 10^{-2}$ to $5 \times 10^{-7}$ and the plasmid from $1030 c p-1$ at frequencies from $3 \times 10^{-1}$ to $1 \times 10^{-6}$. It is assumed from the study of the effects of phage 55 in 1030 that the direction of transfer is from the putative donor to the recipient. The highest frequencies were found when the recipient was lysogenic for phage $55,88,71$ or 79 and the lowest when lysogenic for phage 52 and $42 \mathrm{E}$ where the transfer frequencies were often only fivefold greater than the frequency of spontaneous mutation to streptomycin resistance in the donor.

The chromosomal genes sn, derived from the element cpns (Lacey, 1979), were transduced to 1030 wild using a mitomycin C-induced lysate of strain 6936cpns as donor. The effect of ultraviolet irradiation on a transducing lysate indicated a probable chromosomal locus for $s n$ in 1030 (data not shown). Incubation of 1030sn with 1030 S and each of the lysogenic recipients did not result in transfer in any mixture [selection was made with $4.0 \mu \mathrm{g}$ neomycin $\mathrm{ml}^{-1}$ plus $400 \mu \mathrm{g}$ streptomycin $\mathrm{ml}^{-1}$ since $s n$ confers low level (minimum inhibitory concentration $40 \mu \mathrm{g} \mathrm{ml}^{-1}$ ) resistance to streptomycin]. This finding is consistent with the observations that plasmid genes are readily transferred whilst chromosomal genes are not.

\section{Transfer of cp-2 and cp-3 in mixed cultures of derivatives of strain 1030}

When cultures of strains $1030 c p-2$ and $1030 c p-3$ were incubated with each lysogenic recipient, transfer occurred at frequencies similar to or slightly greater than that from $1030 \mathrm{cp}-1$. However, both $1030 \mathrm{cp}-2$ and $1030 \mathrm{cp}-3$ transferred their elements at high frequency $\left(2 \times 10^{-1}\right.$ and $4 \times 10^{-1}$, respectively) to the non-lysogenic strain $1030 \mathrm{~S}$. Cultures of $1030 \mathrm{cp}-2$ and $1030 c p-3$ were inducible with mitomycin $\mathrm{C}$ and ultraviolet light; hence, they probably harbour prophages. These filtrates contained about $10^{-4}$ transducing particles per original donor cell, although no plaque-forming activity has been found after the application of mitomycin C-induced lysates to several indicator cultures including 1030 wild. This prophage might become easily associated with, or even be covalently linked to, the resistance determinants of plasmids $c p-2$ and $c p-3$. If so, it should be possible to transfer this postulated element through several clones of non-lysogenic 1030. Elements $c p-2$ and $c p-3$ each in 10 clones of 1030 were successively transferred (at frequencies of $10^{-1}$ to $10^{-3}$ ) from $1030 \mathrm{~S}$ to $1030 \mathrm{~F}$ and back to $1030 \mathrm{~S}$. Ten derivatives of each of the final resistant recipients (1030S $c p-2$, $1030 \mathrm{~S} c p-3)$ were tested for inducibility to mitomycin $\mathrm{C}$ and ultraviolet light. All were inducible with either agent. Thus, it is believed that linked transfer of plasmid functions and prophage genome has occurred, because the possibility of simultaneous transfer of plasmid and prophage at the high frequencies obtained is extremely unlikely.

The presence of plasmid $c p-1, c p-2$ or $c p-3$ in recipient $1030 \mathrm{~S}$ did not alter the frequency of transfer of plasmids from either 1030(55)tet-r, 1030(55)neo-r or 1030(55)chl-r in mixed cultures (data not shown).

\section{Simultaneous transfer of plasmids}

Cultures were constructed that harboured four plasmids (Table 8) and resistance transfer to recipient $1030 \mathrm{~S}$ was studied. Co-transfer of either neomycin or tetracycline resistance with resistance to $\mathrm{Cd}^{2+}$ occurred from the donor to the recipient as long as the elements $c p-2$ or $c p-3$ were in the donor (Table 8 ). However, examination of five of each of the doubly-resistant recipients revealed that the two genes were subsequently transferred in mixed culture to $1030 \mathrm{~F}$ separately. Thus, it was inferred that coincidental transfer of the two elements had occurred rather than the transfer of single structures. Mitomycin C-induced 
Table 8. Transfer of plasmids in mixed cultures of derivatives of strain 1030

\begin{tabular}{|c|c|c|c|c|c|c|}
\hline \multirow[b]{2}{*}{ Donor } & \multirow[b]{2}{*}{ Recipient } & \multicolumn{5}{|c|}{ Frequency of transfer* of resistance $\dagger$} \\
\hline & & $\mathrm{Cd}^{2+}$ & Tetracycline & Neomycin & $\begin{array}{l}\mathrm{Cd}^{2+} \text { and } \\
\text { tetracycline }\end{array}$ & $\begin{array}{l}\mathrm{Cd}^{2+} \text { and } \\
\text { neomycin }\end{array}$ \\
\hline $1030 \mathrm{cp}-1$ tet-r neo-r chl-r & $1030 \mathrm{~S}$ & 0 & 0 & $\mathbf{0}$ & 0 & 0 \\
\hline 1030 cp-2 tet-r neo-r chl-r & $1030 \mathrm{~S}$ & $4 \cdot 7 \times 10^{-1}$ & $2 \cdot 0 \times 10^{-3}$ & $2 \cdot 9 \times 10^{-4}$ & $9 \cdot 1 \times 10^{-6}$ & $3.0 \times 10^{-7}$ \\
\hline $1030 c p-3$ tet $-r$ neo-r chl-r & $1030 \mathrm{~S}$ & $1.9 \times 10^{-1}$ & $6 \cdot 2 \times 10^{-3}$ & $6.8 \times 10^{-4}$ & $2 \cdot 2 \times 10^{-6}$ & $6 \cdot 3 \times 10^{-7}$ \\
\hline
\end{tabular}

lysates of culture $1030 \mathrm{cp}-2$ neo-r tet-r chl-r were used to transduce these resistances to 1030 wild. Transduction of $c p-2$ (at a frequency of about $10^{-4}$ per original donor cell) was observed, with no evidence of simultaneous transduction of other resistances. Similarly, after phage 88 was propagated on the culture $1030 \mathrm{cp}-2$ neo-r tet-r chl-r each resistance was transduced separately (frequency about $10^{-4}$ ), but no cotransduction was detected.

Transduction of tetracycline resistance from lysogenic derivatives of strain 1030

Mitomycin C-induced lysates of nine tetracycline-resistant lysogenic derivatives of strain 1030 and also non-lysogenic 1030tet-r were examined for their ability to transduce tetracycline resistance to 1030 wild. The cultures lysogenic for phages $\mathrm{C}, 54,96,71,42 \mathrm{E}, 88,55$ and 95 transduced tetracycline resistance at frequencies from $6.0 \times 10^{-4}$ for phage 96 to $3.8 \times 10^{-7}$ for phage 88 and $1.0 \times 10^{-8}$ for phage $42 \mathrm{E}$. Lysates of cultures $1030(77)$ tet- $r$ did not transduce $\left(<10^{-9}\right)$. Culture 1030tet- $r$ was non-inducible and its filtrate after mitomycin $\mathrm{C}$ treatment could not transduce tetracycline resistance. Thus, several phages in addition to those traditionally considered to be transducing phages can mediate this type of resistance transfer.

\section{DISCUSSION}

In addition to the well-known involvement of bacteriophages in transformation and in transduction using cell-free preparations, the presence of bacteriophages in $S$. aureus can affect the occurrence and frequency of transfer of plasmids in mixed cultures in at least five ways. Normal lysogeny in either the donor or the recipient, or the presence of bacteriophage genes as part of a plasmid $(c p-2, c p-3)$ in the donor, can increase the frequency of spontaneous transfer in mixed cultures, and the presence of some bacteriophages in either donor or recipient can inhibit transfer. Evidence is presented here that spontaneous transfer in mixed cultures has characteristics distinct from that mediated by conventional transduction, specifically: (i) transfer of resistance can occur from a non-lysogenic donor to a recipient; (ii) a culture lysogenic for one phage [1030(52)] cannot acquire resistance by transduction with phage $C$ but can acquire resistance following incubation in mixed culture with $1030(C)$ derivatives; (iii) the frequency of transfer can be as high as $7 \cdot 0 \times 10^{-1}$ (resistant recipients/ donor) following mixed culture incubation; (iv) little spontaneous phage activity is seen following subculture of mixed broth cultures to solid agar although a high frequency of transfer may have occurred; (v) filtrates of mixed cultures may not contain agents able to promote transfer, although transfer occurs between cells at high frequency.

However, the transfer of resistance from culture 13136 to 1030 in mixed cultures could well be by spontaneous transduction, i.e. the plasmids are enclosed in phage protein, although it is impossible to establish this with absolute certainty. Similarities between transfer in mixed culture and transfer by transduction are also seen in the requirement for low concentrations (about 0.001 to $0.01 \mathrm{M}$ ) of $\mathrm{Ca}^{2+}$ or $\mathrm{Mg}^{2+}$ and the higher frequencies of transfer of plasmid genes compared with transfer of chromosomal genes. 
The elements $c p-2$ and $c p-3$ can effect their own transfer at high frequency, probably by becoming part of a phage genome. These elements arose spontaneously when cells of strain M7 were incubated with strain 6936 (Lacey, 1979). Strain M7 probably contains defective bacteriophage(s) (Lacey \& Stokes, 1979) and the element cpns may well have recombined with this genome to produce the elements $c p-2$ and $c p-3$. These elements are not identical since the amount and control of beta-lactamase determined by them differs (V. T. Rosdahl, personal communication). Although their origin cannot be identified with certainty, the presence of elements determining production of beta-lactamase that can effect their own transfer among strains of $S$. aureus is a considerable threat to antimicrobial therapy. It is notable that the element cpns passes spontaneously between clones of culture 6936 at a higher frequency than do $c p-1, c p-2$ or $c p-3$ (Lacey, 1979, and unpublished observations). Possibly, phages in this culture inhibit the transfer of the latter elements to a greater extent than that of cpns.

It is suggested that the plasmid transfer in mixed cultures from a non-lysogenic donor be described as 'phage-mediated conjugation' since direct cell-to-cell contact seems necessary, although the mechanism is obscure. The staphylococcal cell surface requires further study before detailed understanding of this mechanism is obtained. However, the occurrence of two types of gene transfer in this species, in addition to transformation, could account for the discrepancies mentioned in the Introduction. Because of the requirement for a high molarity of $\mathrm{Ca}^{2+}(0 \cdot 1 \mathrm{M})$ and the absence of DNAase for optimal frequencies, gene transfer by transformation in staphylococci is probably not common in nature. Spontaneous transfer by generalized transduction in vivo is also of limited potential because of the probable death of the donor cell and some recipients. In contrast, resistance transfer by phagemediated conjugation would be expected to occur without a requirement for the death of the donor cell. Most staphylococcal plasmids exist in multiple copies in the cell (Chopra et al., 1973). It is therefore possible for a donor cell to transfer several of its copies of a particular plasmid to different recipient cells with a total increase in the numbers of resistant cells in the culture.

Resistance to neomycin and tetracycline can be co-transferred with the penicillinase plasmids $c p-2$ or $c p-3$, although each element is subsequently transferred individually from the recipient.

The presence of phage genome could cause cell-to-cell adhesion, presumably by altering surface proteins, and occasionally two plasmids pass to the recipient coincidentally. Thus, the elements $c p-2$ and $c p-3$ not only effect their own transfer, but can mediate transfer of plasmids coding for neomycin or tetracycline resistance. The high frequencies of transfer of plasmids obtained when non-lysogenic derivatives of 1030 are used as donors are probably atypical since most staphylococci are lysogenic.

An incidental finding during these studies has been that lysogenic derivatives of strain 1030 and, to a lesser extent, plasmid-containing cells are consistently more sensitive to both sulphadiazine and trimethoprim than the corresponding wild strain. The presence of the additional cellular DNA may cause an increase in sensitivity to these agents although the mechanism is not established. Lysogeny might increase the efficiency of thymineless death provoked by these agents. These findings could be of value for the isolation of non-lysogenic bacteria or in the detection of plasmid elimination, and might be relevant to the use of these agents therapeutically.

I thank Evelyn Lewis and Peter Bennett for electron microscopy, and V. Lord and S. Russell for technical assistance.

\section{REFERENCES}

Chopra, I., Bennett, P. \& Lacey, R. W. (1973). A variety of staphylococcal plasmids present as multiple copies. Journal of General Microbiology 79, 343-345.
Devoret, R. \& Blanco, M. (1970). Mutants of Escherichia coli $\mathrm{K} 12(\lambda)^{+}$non-inducible by thymine starvation. Molecular and General Genetics 107, 272-280. 
LACEY, R. W. (1971a). High-frequency transfer of neomycin resistance between naturally-occurring strains of Staphylococcus aureus. Journal of Medical Microbiology 4, 73-84.

LACEY, R. W. (1971b). Transfer of tetracycline resistance between strains of Staphylococcus aureus in mixed cultures. Journal of General Microbiology 69, 229-237.

LACEY, R. W. (1972). Genetic control in methicillinresistant strains of Staphylococcus aureus. Journal of Medical Microbiology 5, 497-508.

LACEY, R. W. (1975). Antibiotic resistance plasmids of Staphylococcus aureus and their clinical importance. Bacteriological Reviews 39, 1-32.

LACEY, R. W. (1979). An unusual element from Staphylococcus aureus. Journal of Medical Microbiology 12, 311-320.

LACEY, R. W. \& Grinsted, J. (1973). Genetic analysis of methicillin-resistant strains of Staphylococcus aureus: evidence for their evolution from a single clone. Journal of Medical Microbiology 6, 511-526.

LACEY, R. W. \& Stokes, A. (1979). Studies on recently isolated cultures of methicillin-resistant Staphylococcus aureus. Journal of General Microbiology 114, 329-339.

LaNTos, J. (1977). Transfer of erythromycin resistance in Staphylococcus aureus. Acta microbiologica Acad:miae scientiarum hungaricae 24, 107-113.

LINDberg, M. \& Novick, R. P. (1973). Plasmidspecific transformation in Staphylococcus aureus. Journal of Bacteriology 115, 139-145.
Melechen, N. E. (1962). The provocation of an early step of induction by thymine deprivation. Virology 16, 21-29.

Naidoo, J. \& Noble, W. C. (1978). Transfer of gentamicin resistance between strains of Staphylococcus aureus on skin. Journal of General Microbiology 107, 391-393.

Novick, R. P. (1963). Analysis by transduction of mutations affecting penicillinase formation in Staphylococcus aureus. Journal of General Microbiology 33, 121-136.

Novick, R. P. (1967). Properties of a cryptic highfrequency transducing phage in Staphylococcus aureus. Virology 33, 155-166.

Novick, R. P. \& Morse, S. I. (1967). In vivo transmission of drug resistance factors between strains of Staphylococcus aureus. Journal of Experimental Medicine 125, 45-59.

Pattee, P. A. \& Baldwin, J. N. (1961). Transduction of resistance to some macrolide antibiotics in Staphylococcus aureus. Journal of Bacteriology 84, 875-881.

RichmoND, M. H. (1969). Extrachromosomal elements and the spread of antibiotic resistance in bacteria. Biochemical Journal 113, 225-235.

Robinson, M. K., BENNETT, P. M. \& RichMond, M. H. (1977). Inhibition of TnA translocation by TnA. Journal of Bacteriology 129, 407-414.

WitTE, W. (1977). Transfer of drug resistance plasmids in mixed cultures of staphylococci. Zentralblatt für Bakteriologie, Parasitenkunde, Infektionskrankheiten und Hygiene 237, 147-159. 